\title{
Nivel de conocimiento de protocolos de primeros auxilios en docentes de educación inicial y preparatoria de instituciones educativas de la ciudad de Quito
}

Level of knowledge of first aid protocols in initial and preparatory education teachers belonging to different educational institutions in Quito city

\author{
MARÍA TERESA MURILLO LOJÁN (D) ERIKA MARLENE VARGAS FUERTES (D), \\ JANETH DEL PILAR LÓPEZ SEVILLA(*)(D) \\ Universidad de las Fuerzas Armadas ESPE, \\ Departamento de Ciencias Humanas y Sociales, \\ Av. General Rumiñahui s/n y Ambato \\ Sangolquí - Ecuador
}

*janethlopezs22@hotmail.com

\section{RESUMEN}

Los protocolos de primeros auxilios dentro de las instituciones educativas tanto públicas, privadas o municipales de la ciudad de Quito, relacionados a la Educación Inicial y Preparatoria, son temas que deben ser prioritarios, con énfasis en la preparación de las/os docentes, en razón de ser ente principal de cuidado y enseñanza de los niños/as. Por tanto, es necesario que estén calificados para brindar la asistencia a sus estudiantes ante algún accidente dentro de las instalaciones de los centros infantiles. Propósito: Valorar los conocimientos de los protocolos de primeros auxilios en autoridades y docentes de Educación Inicial y Preparatoria de los establecimientos educativos en Quito. Sujetos y métodos: Estudio multicéntrico, observacional, transversal de 
una cohorte. Población: 54 docentes y autoridades de educación inicial y preparatoria de la ciudad de Quito, de agosto a septiembre de 2020. Resultados: En cuanto a capacitación de primeros auxilios, el $80 \%$ de educadores encuestados recibieron preparación en el tema; mientras el 20 \% restante no recibieron capacitación de primeros auxilios durante su formación. En ese contexto buscó conocer si los docentes poseen conocimiento de primeros auxilios, resultando que el $98 \%$ sabía a qué se refiere el término; mientras que el $2 \%$ no conocían. Conclusión: Se concluyó que la capacitación, teórica y práctica, de primeros auxilios es una herramienta fundamental para precautelar la integridad física y psicológica de las/os niños.

Palabras claves: primeros auxilios, preparación docente, educación inicial, preparatoria, accidente.

\section{ABSTRACT}

Background: First aid protocols within public, private, or municipal educational institutions of the city of Quito, which are related to Initial and Preparatory Education, are issues that should be prioritized with emphasis on the training of teachers since these people are the ones who teach and take care of children. Therefore, it is necessary that instructors need to be qualified to provide some aid to their students in the event of an accident could take place inside the children's centers. Purpose: To assess the authorities and teachers' knowledge of the first aid protocols and who belong to the Initial and Preparatory Education of the different educational institutions in Quito. Subjects and methods: This is a Multicenter, observational, and cross-sectional study of a specific cohort. Population: 54 teachers and authorities of initial and preparatory education of the city of Quito. Period: from August to September 2020. Results: Regarding first aid training, $80 \%$ of educators surveyed received some training on the subject; while the remaining $20 \%$ did not receive any first aid training during their preparation as teachers. In this context, it was sought to know if teachers have some knowledge of first aid, resulting that $98 \%$ knew what the term refers to, while $2 \%$ did not know anything. Conclusion: It was concluded that the theoretical and practical first aid training is a fundamental tool to protect children's physical and psychological integrity.

Keywords: first aid, teacher's preparation, initial education, preparatory, accident.

\section{INTRODUCCIÓN}

En Ecuador de acuerdo a los Registros Administrativos 20192020 del Ministerio de Educación (2020), se estima que existen 8230 instituciones que ofertan los niveles de Educación Inicial y Preparatoria. De las cuales, 1077 se encuentran en la ciudad de Quito, laboran un aproximado de 7191 docentes y directivos.

En lo que se relaciona con los estudiantes, se estima que por aula existe un mínimo de 15 y un máximo de 25.

La investigación está dirigida a demostrar la importancia de aplicar los protocolos de primeros auxilios ante una emergencia o situación de riesgo que puede presentarse dentro del aula o en las instalaciones. 
Dentro de la normativa de Educación Inicial del país, se fomenta la participación de las instituciones educativas en la organización de diferentes simulacros que estén orientados a desastres naturales como temblores, terremotos o incendios. Fernández \& Fernández (2015) refieren "en el caso de los niños, los accidentes son mucho más frecuentes que en los adultos, debido a que los niños no tienen noción del peligro" p.12. Los autores mencionan que se debe tener importante consideración para evitar accidentes de acuerdo a la edad de los niños y el espacio en el que se encuentran.

Singh et.al (2016) hacen referencia a la OMS mencionando que los accidentes son acontecimientos imprevistos que generan daños independientemente de la voluntad humana, es decir, son provocados por agentes externos y causan lesiones físicas y/o psicológicas. Al hacer referencia a eventos imprevistos, estos pueden ocurrir en cualquier lugar: dentro del hogar, parques de recreación, centros comerciales etc., y por supuesto dentro de las aulas de educación inicial y preparatoria. Estos eventos fortuitos condicionan a que las causas y consecuencias sean diversas, desde un accidente causado por una enfermedad hasta una caída durante un momento lúdico entre compañeros, subiendo o bajando las gradas, un tropezón al correr, entre otros. Varios de los tipos de accidentes son dados dentro de instituciones y pueden ser leves, moderados o graves. Adicional a esto, el Ministerio de Educación del Ecuador (2016) menciona que los niños menores de seis años se encuentran expuestos a los siguientes tipos de accidentes: asfixia, estado de shock, heridas, fracturas abiertas o cerradas, luxaciones, quemaduras, convulsiones, mordeduras de animales o de compañeros, intoxicación, electrocución, desmayos, politraumatismos, paro cardiorrespiratorio y deshidratación.
Dentro de los primeros auxilios es necesario reconocer que los métodos aplicados varían de acuerdo a la edad y condición en la que se encuentre el niño. En este contexto, Estrada, Gutiérrez \& Hernández (2017), refieren que existen métodos como la reanimación cardio respiratoria, que debe ser aplicada con conocimientos técnicos previos para no generar mayor daño, por ello destacan la importancia de las capacitaciones a los docentes de nivel escolar.

Frente a la diversidad de causales y consecuencias, es importante que el docente esté preparado para atender cualquier tipo de acontecimiento que implique poner en marcha un protocolo básico de primeros auxilios. Saber cómo actuar ante cada tipo de emergencia debería convertirse en la base primordial de la formación profesional de los docentes, Martín (2015) refiere que "la solución inmediata ante un accidente son los primeros auxilios" p. 86. Considerando que los niños y niñas, pasan gran parte de su tiempo en los establecimientos educativos, estas soluciones inmediatas deben ser recibidas por parte de sus docentes, en este contexto la autora afirma que: "la educación sobre principios básicos de primeros auxilios de la población tiene un gran impacto sobre la mortalidad y morbilidad de las enfermedades no transmitibles" p. 89

Bajo este contexto, el entorno educativo se convierte en uno de los principales lugares donde el conocimiento de primeros auxilios es fundamental, pues en las escuelas los niños comparten gran parte de su tiempo, esto los vuelve más propensos a sufrir cualquier tipo de accidente dentro y fuera de las aulas. El mismo hecho de que un niño o niña se encuentre en formación, permite que su exploración, deseos de aprendizaje constante y falta de 
medición del peligro, los exponga a sufrir cualquier tipo de accidente, puesto que, es la imprudencia de su edad lo que provocan acciones que pueden terminar en una tragedia.

Cuando revisamos bibliografía en afinidad a la preparación en salud de los docentes, nos damos cuenta que existen varios estudios al respecto, Marchena (2018) menciona a "los españoles como los ciudadanos peor preparados de Europa para hacer frente a una situación que requiera proporcionar primeros auxilios". Asimismo, Rodríguez (2020) refiere que un tercio la población española reconoce no sentirse para nada seguro en caso de tener que brindar primeros auxilios.

En un contexto más cercano es importante mencionar a Quishpe (2018), quien hace mención a un estudio de investigación en Perú, donde se menciona que: "el 38,9\% de los docentes presentan un conocimiento regular sobre los primeros auxilios, seguido del $36,1 \%$ con un conocimiento deficiente y el $25 \%$ con un conocimiento bueno". Finalmente, es importante considerar las cifras en nuestro país, que no están alejadas de la realidad de Latinoamérica y España. Chachapoya (2019) hace referencia a una investigación realizada en el período octubre 2016 a febrero 2017 en la ciudad de Manta, en donde se menciona que los conocimientos de primeros auxilios que se recibe en la formación para docentes, si bien es teórica, debe ir de la mano con la práctica, lo cual les permitirá actuar de una mejor manera al momento de atender una emergencia dentro del aula. Finalmente, dentro de las estadísticas debemos mencionar el estudio realizado por Salvatierra (2017) quien alude que, debido al desconocimiento de primeros auxilios por parte de los docentes de Guayaquil, los niños que sufren accidentes escolares ingresan gravemente a los centros de atención hospitalaria, situación que debería ser de prioritaria atención desde el Ministerio de Educación del Ecuador.

Con base en los datos estadísticos presentados en las investigaciones anteriores, se considera necesario realizar una investigación direccionada a identificar cuanto conocen los docentes de educación inicial y preparatoria de la ciudad de Quito sobre los primeros auxilios, se debe analizar si durante la formación del docente se han implementado conocimientos básicos que les permita actuar durante una emergencia, - determinar si por iniciativa propia el docente adquiere conocimientos mediante capacitaciones y formación continua que les permita actuar con premura ante una emergencia. Estos factores son de vital importancia en nuestra investigación, sin embargo, consideramos primordial conocer cuán necesaria es una guía que destaque el valor de conocer los parámetros de primeros auxilios necesarios de acuerdo a las necesidades y características de los niños y niñas.

\section{PLANTEAMIENTO DEL PROBLEMA}

Según la OMS (2016) aproximadamente 5,6 millones de niños murieron antes de cumplir cinco años. Esto se traduce en 15000 muertes de menores de cinco años por día, siendo las principales causas de muerte infantil: enfermedades crónicas, anomalías congénitas y lesiones de distintos tipos como accidentes de tránsito, ahogamiento, quemaduras y caídas. Este tipo de lesiones según la OMS, hasta septiembre 2020 están entre las tres principales causas de muerte y generadoras de discapacidad 
en niños de 5 a 15 años. Respecto al contexto escolar, en el resumen mundial sobre la prevención de las lesiones en los niños por la OMS (2020), se destaca que en Polonia, en 202 escuelas se presentaron 3274 accidentes escolares, de los cuales 36.6 $\%$ ocurrieron en el momento de descanso, $33.2 \%$ ocurrieron en la clase de educación física, y el $29.7 \%$ ocurrieron en el patio de recreo.

Una realidad con estadísticas más alarmantes está en el contexto latinoamericano; Socota (2014) refiere que, en Colombia, en el año 2014, ocurrieron más de 1400 accidentes en los colegios oficiales de Bogotá. Entre ellos, un niño de 11 años perdió la visibilidad del ojo izquierdo por un juego en el salón de clases y un niño de 4 años de un jardín infantil sufrió de asfixia con una ficha de parqués. En este estudio los docentes practicaron maniobras inadecuadas como el ponerlo de cabeza, tomarlo de los pies y comenzaron a darle palmadas en la espalda; por lo que, practicarle una maniobra inadecuada a un niño desencadena consecuencias fatales.

En Ecuador, la realidad es similar en cuanto a accidentes escolares, sin embargo, se desconoce con exactitud una cifra y cómo son atendidos. Las cifras que se mencionan son casos reportados por periódicos o medios de comunicación. En el 2019, según refiere Trujillo (2020), una bebé de tan solo nueve meses de edad fallece a causa de un ahogamiento, que de manera lamentable terminó con la vida de la menor, puesto que las docentes y encargadas del centro del desarrollo infantil desconocían qué parámetros seguir en caso de esta emergencia. Chachapoya (2019) en su investigación dentro de la Unidad Educativa Oswaldo Lombeyda en el año 2018, relata accidentes en los primeros años de educación general básica. Un niño entre 5 a 6 años de edad, mientras ingería una fruta, sufrió atoramiento y asfixia. $Y$ en otro caso, en el momento de receso un niño se encontraba jugando en el patio de la institución y en uno de los juegos el niño sufrió un fuerte golpe con el columpio, lo que le generó un trauma en la zona interior de su cabeza. En ambos casos, las autoridades mencionan que las docentes encargadas no sabían que protocolos de primeros auxilios seguir; por lo que fue necesario que el personal médico se hiciera cargo de la situación.

Cuando hablamos de primeros auxilios, es importante conceptualizar previamente el término, Martín (2015) hace referencia a los conocimientos de técnicas e instrumentos que son necesarios para atender una emergencia en cualquier ámbito donde se encuentre en riesgo la integridad de una persona. Otros autores como Estrada et. al (2017) definen a los primeros auxilios como los cuidados inmediatos, adecuados y provisionales brindados a las personas accidentadas o con enfermedades de aparición súbita antes de la llegada de profesionales especializados, o de la respectiva atención en un centro asistencial.

La Empresa Pública de Emergencias Sanitarias Española (2019) menciona que dentro de una emergencia donde sean necesarios los primeros auxilios, se debe tomar en cuenta los siguientes principios para brindar ayuda en caso de una situación de riesgo:

\section{- Evaluar la situación de lo que está sucediendo previo}

al actuar. Consiste en analizar qué medidas de seguridad son necesarias implementar para atender al accidentado. 
Nivel de conocimiento de protocolos de primeros auxilios en docentes de educación inicial y preparatoria de instituciones educativas de la ciudad de Quito - María Teresa Murillo, Erika Marlene Vargas, Janeth del Pilar López • VÍNCULOS-ESPE (2021) VOL.6, No.3: 47-66

- Evaluar la situación en la que se encuentra el niño. Con el objetivo de comprobar niveles de conciencia mediante la toma de signos vitales. Lo que permitirá evidenciar el grado de estabilidad y que tan manejable es la situación.

\section{- Conocer los números de los servicios de emergencia o} hospitales cercanos, para que se pueda socorrer de mejor manera al herido con asistencia especializada en prontitud.

Es importante considerar que en todo momento se debe estar junto a la víctima, puesto que el accidentado necesitará de constante apoyo en caso de estar consiente.

\section{Preparación y formación docente en primeros auxilios}

Considerando la importancia de brindar los primeros auxilios, es importante discurrir en la idea que, dentro de la formación y preparación docente, se debe contemplar de manera prioritaria el trabajar en temas de primeros auxilios. Delgado \& Espinoza (2019) en su investigación, refieren que, en países desarrollados como Estados Unidos, se capacita permanentemente a los docentes ante posibles accidentes que se pueden producir dentro de las aulas; dentro de la malla curricular se toma en cuenta una materia llamada Educación para la Salud, que los prepara en el tema de primeros auxilios, y una vez que estos docentes ya estén ejerciendo, son capacitados de manera constante.

En cuanto a países latinoamericanos, Delgado \& Espinoza (2019), acotan que, si bien en algunos casos la preparación docente en temas de primeros auxilios es tomada en cuenta, muchos de sus contenidos no son actualizados ni puestos en práctica constante.
En Ecuador no se cuenta con una formación extensa en estos temas de salud, es decir, no se presta mucha atención a la Educación para la Salud dentro de lo que es la formación docente.

A pesar de que existan manuales que expliquen de manera general o específica las formas de actuar en caso de una emergencia, en ciertas instancias en el momento que ocurre un accidente en los períodos de clases, la mayoría de los docentes de los Centros de Educación Inicial (C.E.I.), se sienten inseguros para brindar ayuda inmediata a un niño o niña accidentando. Hay consideraciones prioritarias dentro del manual de primeros auxilios, una de vital importancia es la presencia del botiquín, Delgado \& Espinoza (2019) refieren en su investigación que existen instituciones educativas que cuentan con un botiquín, sin embargo, los recursos dentro de él son insuficientes y sumada a esta realidad está el desconocimiento frente al uso inmediato y adecuado del mismo. En este contexto, se debe priorizar que el personal docente se encuentre en constantes capacitaciones, con primordial atención en la implementación del plan P.A.S, Maza \& Maza (2019), describen dentro de su guía de primeros auxilios la importancia de las conductas dirigidas a ejecutar el plan de Proteger, Avisar y Socorrer, método que se detallará en un posterior apartado. Es así como la atención y actuación del docente permitirá mediante una serie de medidas preventivas reforzadas con el conocimiento teórico y práctico salvaguardar la vida de sus alumnos.

\section{Primeros auxilios en educación inicial y preparatoria}

Los centros de educación inicial son espacios donde puede existir la posibilidad de que se produzcan accidentes, por ende, 
los docentes deben estar capacitados y tener conocimientos sobre qué hacer en esos casos. Fernández y Fernández (2015) mencionan que "La edad donde son más frecuentes los accidentes infantiles es entre los 2 y 3 años y entre los 11 y 14, siendo más frecuentes en los niños que en las niñas" (p.16). Por lo que se debe poner especial énfasis en las medidas preventivas y en capacitaciones al personal a cargo de los niños y niñas sobre el tema.

En este aspecto, en Ecuador, Delgado \& Espinoza (2019) relatan en su investigación que el Ministerio de Educación del Ecuador desde el 2016 otorgó a los docentes una guía que les ayuda a conocer cómo prevenir una serie de riesgos y accidentes, tanto dentro como fuera del aula; con un principal objetivo, intervenir en el aula para disminuir las condiciones de vulnerabilidad. El propósito de esta guía, es priorizar la seguridad de cada uno de los agentes educativos, pero sobre todo se pretende precautelar la integridad de los niños y niñas, en temas de seguridad. Para cumplir con estos propósitos, es importante que el docente identifique los factores de riesgo, por ello dentro de la guía se clasifica a los riesgos y accidentes, a los que se encuentra expuestos los pequeños dentro de las instituciones de la siguiente manera:

- Amenazas de origen natural, son todos los accidentes y situaciones cuyo origen sea natural: sismos, terremotos, etc.

- Riesgos asociados a accidentes escolares, encontramos caídas de distintos niveles, golpes, cortes, punzadas, ahogamiento, atragantamiento, electrocución, incendio, explosión, atropellamiento, arrollamiento y choques.
- Riesgos asociados a la violencia social, son peligros a los que se encuentran expuestos los pequeños fuera de las instalaciones de las instituciones, como robo, hurto y secuestro.

La Cruz Roja Ecuatoriana (2017) detalló en uno de sus informes anuales una lista de todos aquellos tipos de accidentes que niños de 0-6 años pueden llegar a sufrir en los entornos educativos, en los que se debería tener la ayuda inmediata de los docentes y autoridades, considerando por ello de importancia el conocimiento en parámetros básicos sobre primeros auxilios, entre los principales accidentes se mencionan:

- Obstrucción de la vía aérea, asfixia: este tipo de riesgo es producido cuando dentro de la garganta, se encuentra un objeto o alimento que produce la obstrucción de la respiración. Las causas son varias y producen en el menor una imposibilidad para respirar por lo que el color de su rostro, labios y uñas, se torna azulado. Para este tipo de emergencias se necesita emplear el RCP (reanimación cardiopulmonar), siempre y cuando se conozca su aplicación de acuerdo a las particularidades del caso.

- Estado de shock: el estado de shock, es la disminución de la oxigenación celular generalizada, que provoca daños en el funcionamiento del organismo. Este estado va a provocar en el niño palidez, temperatura baja, alteración de la conciencia, sudoración, reparación rápida y un pulso débil.

- Heridas: se producen por golpes que ocasionan lesiones en la piel, ocurren con mayor frecuencia por actividades físicas 
que el infante realiza diariamente, estas lesiones pueden ser superficiales como los raspones, o profundas en las que ya se presentan hemorragias. También, puedes ser consideradas como lesiones leves y graves, éstas pueden producirse en extremidades, cara, cabeza, tórax o abdomen.

- Fracturas: se definen como la ruptura de uno o más huesos, producidas por una caída o un golpe demasiado fuerte.

- Luxación: este tipo de lesión es producida por movimientos bruscos que provocan que una articulación se salga de su lugar, para tratar estas luxaciones se necesita de técnicas de inmovilización, dependiendo de la parte del cuerpo afectada.

- Quemadura: son heridas o lesiones que se producen en la piel de cualquier parte del cuerpo del niño. Pueden ser causadas por la exposición al calor extremo como: agua hiviendo, sol, electricidad, radiación o exposición a químicos. Sus niveles son: superficiales o de primer grado, espesor parcial o segundo grado, tercer grado y cuarto grado; de acuerdo a su gravedad.

- Convulsiones: son movimientos incontrolables e involuntarios del cuerpo humano, producidos cuando el cerebro deja de funcionar normalmente. desinfectantes, blanqueadores, monóxido de carbono, gas casero, etc.

- Electrocución: se debe al contacto con aparatos eléctricos, cuyos cables estén expuestos o cuando se colocan objetos dentro de interruptores expuestos.

- Lesiones térmicas: causadas por la exposición excesiva al sol o sobreesfuerzos, en donde el calentamiento del cuerpo produce deshidratación y desmayos.

- Lesiones oculares: causado por cuerpos extraños o heridas, esto puede generar pérdida de la visión.

- Hemorragias: este tipo de accidentes son relativamente comunes y se producen por la salida de sangre descontrolada, pueden ser externas o internas.

Bajo este contexto, podemos concluir que existen muchas formas de que un niño o niña sufra un accidente dentro del ambiente escolar, por lo que es primordial realizar actividades preventivas dentro de los establecimientos que pretendan precautelar la integridad física de los pequeños, y a la par de esta actividad, debe estar la formación en conocimientos sobre primeros auxilios para todo el personal que labora en la institución, en especial docentes y autoridades de cada plantel público y privado.

\section{Primeros auxilios para niños con discapacidad}

El término discapacidad es de uso relativamente nuevo, para Hernández (2015) hace referencia a la evolución de este 
concepto, desde la creencia de ser una deficiencia causada por mala suerte o brujería, hasta llegar a conceptualizarla como una dificultad para realizar las actividades de la vida diaria de manera independiente, debido a las numerosas barreras sociales y físicas. Esta conceptualización ha permitido clasificar a las discapacidades según la OMS (2011) de la siguiente manera:

- Discapacidad sensorial: dentro de este tipo de discapacidades encontramos todas las limitaciones, visuales, auditivas y de lenguaje.

- Discapacidad física: es también conocida como discapacidad motora y es caracterizada por la limitación de las capacidades físicas de la persona. En esta se pueden encontrar la pérdida total o parcial de una extremidad o la inmovilidad de las mismas, debido a un accidente o enfermedad.

- Discapacidad intelectual: es una limitación mental o intelectual que impide el normal relacionamiento con la sociedad, lo que genera mayor deficiencia en la gran mayoría de las áreas de desarrollo de los niños y niñas.

Es importante considerar que un niño o niña sin algún tipo de discapacidad, por su naturaleza curiosa, tiende a no obedecer órdenes o medir el peligro, más aún estos factores se exacerban cuando existe una discapacidad, pues para ellos los accidentes son de más riesgo, razón por la cual al encontrarse en situaciones de peligro pueden llegar a estresarse y no ser capaz de expresar lo que le está sucediendo. Por lo que la actuación del maestro en situaciones de emergencia debe ser enfocada en la particularidad de la discapacidad y en la calma que pueda transmitir al niño o niña.

\section{Primeros auxilios para niños con enfermedades catastróficas}

La OMS (2020) define a las enfermedades catastróficas como aquellas que se caracterizan por su larga duración y por lo general, de progresión lenta, entre las más comunes encontramos malformaciones cardíacas, infartos, cáncer, enfermedades respiratorias, enfermedades que causan altas tasas de mortalidad en el mundo, alterando el estado de salud y la convivencia social de las personas. El Ministerio de Salud Pública del Ecuador, según refiere Cagua (2019), define a las enfermedades catastróficas, raras o huérfanas, como aquellas patologías de curso crónico que suponen alto riesgo y cuyo tratamiento es de alto costo económico e impacto social, mismas que pueden llegar a ser prolongadas o permanentes.

Siendo esta una realidad mundial y considerando que estas enfermedades no se pueden prevenir y tampoco tiene un rango de edad para aparecer, dentro de las aulas de educación inicial y preparatoria, en cualquier momento, los docentes pueden llegar a necesitar asistir a niños cuyas enfermedades requieren de una atención prioritaria y oportuna. Por ello, Páez (2015), destaca la importancia de la actuación de los docentes contemplando las siguientes características: maximizar la capacidad del niño para salir bien en la escuela, comunicación entre la casa, el hospital y la escuela, saber si la enfermedad puede afectar al niño en la clase, como enfrentarse con tal enfermedad y las consecuencias 
del tratamiento relacionadas con la educación. De esta forma se estará preparado y prevenido, pues es importante conocer que enfermedad padece el alumno y cómo se debe actuar frente a una emergencia.

\section{Parámetros básicos de primeros auxilios en educación inicial y preparatoria}

Oña (2016) define a los primeros auxilios en el contexto escolar, como todas aquellas acciones que los docentes llevan a cabo luego de haberse producido un suceso que dé como resultado una lesión, de manera de que el estado del niño no empeore, hasta que se pueda trasladar a la víctima a un hospital o centro médico más cercano. Por lo que, dentro de lo que a parámetros de primeros auxilios se refiere, es necesario conocer aspectos como:

- Prevenir al máximo que cualquier tipo de accidente dentro de las instalaciones de las unidades educativas o en las aulas.

- Identificar números de emergencia, estos permitirán a los docentes comunicarse cuanto antes con el personal especializado, para que guíen a los docentes en las actuaciones determinada emergencia.

En caso que los docentes se enfrenten a una emergencia, se recomienda mantener la calma, identificar el tipo de lesión al que se enfrenta, evaluar la condición en la que se encuentra el niño o niña, tranquilizarlo, determinar el nivel de conciencia y actuar de acuerdo a la emergencia.

\section{Parámetros de primeros auxilios para niños con discapacidad y enfermedades crónicas}

Al tratarse de una educación inclusiva en todos los ámbitos, se debe contemplar que dentro de las aulas los grupos de estudiantes son variados. En función de ello, dentro de la LOEI (2015), cuyas siglas responden a la Ley Orgánica de Educación Intercultural Bilingüe, en su artículo 2 Principios, se destaca la atención e integración prioritaria y especializada de las niñas, niños y adolescentes con discapacidad y enfermedades catastróficas. Es decir, el docente debe considerar las particularidades de cada caso, siendo de atención prioritaria los niños con discapacidad y enfermedades catastróficas. Maza \& Maza (2019), proponen el método PAS, el cual consiste en lo siguiente:

- Proteger: resguardando del peligro al accidentado y a nosotros mismos, con la idea de autoprotección que permite brindar ayuda a los demás.

- Avisar: sin dejar solo al accidentado, llamar a emergencias y brindar información exacta sobre motivo de la llamada, estado de conciencia de los afectados, la ubicación del siniestro, número de afectados y tipo de accidente.

- Socorrer: ayudar a las víctimas mientras llega el personal de emergencia, sin duda esta es la parte más importante, en la que se puede garantizar la vida de los afectados, siempre y cuando se realice de manera oportuna y en conocimiento previo de la técnica que se utilice. 
Previo a la aplicación de este método, se debe considerar siempre realizar una evaluación inicial rápida en la que se debe constatar el estado de conciencia y signos vitales, identificar el objetivo de atención, y ejecutar el procedimiento de acuerdo a la rápida identificación del problema. Esta planificación permitirá ayudar mientras llega el equipo médico al lugar de la emergencia.

\section{Botiquín de primeros auxilios}

Según refiere la Cruz Roja Ecuatoriana (2017), el botiquín de primeros auxilios es un recurso básico para salvar vidas, ya que contiene los elementos necesarios para atender a las víctimas de accidentes o de enfermedades repentinas. Este constituye un elemento primordial para la atención de emergencias en todos los ámbitos. Por lo que, contar dentro de las aulas con esta herramienta es indispensable para atender emergencias para que el botiquín sea de utilidad, es necesario que cuente con los elementos necesarios para salvaguardar la integridad de las personas como: guía telefónica de centros médicos, hospitales cercanos o de emergencia, curitas, gasas, algodón, guantes quirúrgicos, mascarillas quirúrgicas, abate lenguas, vendas de varios tamaños, alcohol antiséptico, agua oxigenada, paracetamol, termómetro, férulas, vendajes triangulares, pinzas, esparadrapo, y apuntes de alergias o enfermedades de los niños, tipo de sangre y medicamentos que usan. Por lo que la presencia del botiquín debe ser por aula, ya que debe tener consideraciones particulares de cada alumno y el docente debería tener presente las novedades en cuanto a salud de cada uno de sus estudiantes.

\section{MÉTODOS}

\section{Diseño del estudio}

Es un estudio multicéntrico, observacional, transversal de una cohorte.

\section{Sujetos}

Se realizó encuestas a 54 docentes y directivos de centros de desarrollo infantil de carácter público, privado y municipal de la Ciudad de Quito que trabajan con niños y niñas de 0-6 años.

\section{Criterios de inclusión, exclusión y eliminación}

\section{Criterios de inclusión generales}

1. Docentes de Maternal, Inicial I, Inicial II y Preparatoria.

2. Directivos de centros de desarrollo infantil e instituciones públicas, privadas y municipales.

3. Docentes de niños con discapacidad.

4. Docentes de ambos sexos.

\section{Criterios de exclusión}

1. Docentes de educación básica, bachillerato y universidades.

2. Docentes que no ejercen su labor

\section{Criterios de eliminación}

1. Docentes que rechacen la participación en el estudio. 
Nivel de conocimiento de protocolos de primeros auxilios en docentes de educación inicial y preparatoria de instituciones educativas de la ciudad de Quito - María Teresa Murillo, Erika Marlene Vargas, Janeth del Pilar López • VÍNCULOS-ESPE (2021) VOL.6, No.3: 47-66

\section{Criterios éticos}

El instrumento aplicado fue en base a la encuesta de Gómez Sanabria, H. (2015), modificada y adaptada a la realidad educativa actual, revisada y validada para su aplicación. Previo a la recolección de datos se contó con el consentimiento de los participantes, lo que permitió continuar con el estudio.

\section{Análisis estadístico}

Se creó una base de datos en el software Excel 2016, para depuración, análisis y transferencia al software SPSS para Windows versión 22.0. Las variables cualitativas se reportan con sus frecuencias absolutas y relativas (Porcentajes). Para todas las comparaciones se consideran significativos valores inferiores al $5 \%(p<0,05)$. Los análisis y gráficos se realizaron usando el programa estadístico " $r$ ".

\section{RESULTADOS}

La Tabla 1 muestra que, dentro del grupo del grupo de 54 encuestados para la presente investigación, el $78 \%(n=42)$ fueron docentes de los cuales el $24 \%$ laboran en instituciones públicas, el $45 \%$ en instituciones municipales y el $31 \%$ en instituciones privadas. De la misma manera se tomó en cuenta a las autoridades de distintas instituciones a las cuales corresponde el $22 \%(n=12)$ del grupo total, de los cuales el $50 \%$ laboran en instituciones públicas, el $25 \%$ en municipales y el otro $25 \%$ en privadas.
Tabla 1

Distribución de las características de docentes y autoridades encuestadas

\begin{tabular}{ccccccc} 
Variable & \multicolumn{2}{c}{ Docentes } & \multicolumn{2}{c}{ Autoridades } & \multicolumn{2}{c}{$\begin{array}{c}\text { Población } \\
\text { acumulada }\end{array}$} \\
& Nro & $\%$ & Nro & $\%$ & Nro & $\%$ \\
\hline Públicas & 19 & 35 & 6 & 50 & 25 & 46 \\
\hline Municipales & 13 & 24 & 3 & 25 & 16 & 30 \\
\hline Privadas & 10 & 19 & 3 & 25 & 13 & 24 \\
\hline Total & 42 & 78 & 12 & 100 & 54 & 100 \\
\hline
\end{tabular}

Nota. Obtenido de encuesta.

Tabla 2

Aula en la que dictan clases los docentes encuestados

$$
\begin{array}{cc}
\text { Docentes } \\
(\mathrm{n}=42)
\end{array}
$$

Nivel en el que dan clases $(\mathbf{n}, \%)$

\begin{tabular}{ccc} 
Maternal & 14 & 33 \\
\hline Inicial I & 2 & 5 \\
\hline Inicial II & 5 & 12 \\
\hline Preparatoria & 19 & 45 \\
\hline Inicial I y II & 1 & 3 \\
\hline Inicial I y II , Preparatoria & 1 & 2 \\
\hline
\end{tabular}

Nota. Obtenido de encuesta. 
La Tabla 2 presenta la distribución de los docentes que corresponden al $78 \%(n=42)$ del grupo total de encuestados. Según el nivel en el que laboran, el 33 \% trabajan en el área de Maternal, el $5 \%$ en Inicial I, el $5 \%$ en Inicial II, el $45 \%$ en Preparatoria, el $3 \%$ trabaja con niños tanto de Inicial I y II y el 2 $\%$ restante trabaja con un grupo de niños más variado en edad, puesto que el grupo es de Inicial I, Inicial II y Preparatoria.

\section{Figura 1}

Porcentaje promedio de conocimientos de primeros auxilios, en función de preguntas teóricas

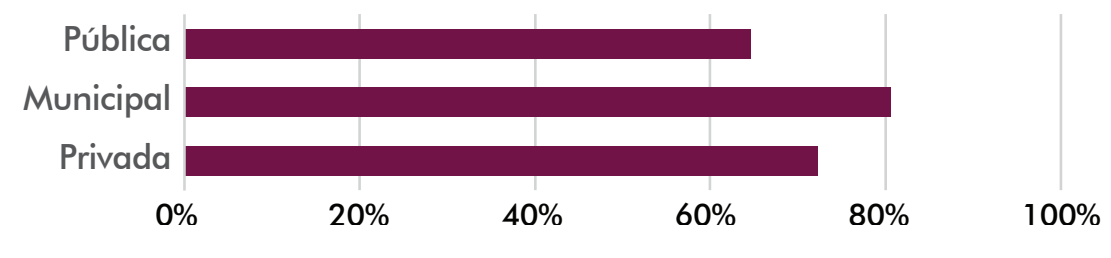

Nota. Obtenido de encuesta.

La Figura 1 muestra, con respecto a interrogantes de carácter teórico, los conocimientos en primeros auxilios por parte de docentes y autoridades de las distintas instituciones. Dentro de este estudio, el 98\% contestó afirmativamente a la interrogante y tan sólo un $2 \%$ dio una contestación negativa a la misma. En función a la interrogante anterior, se planteó si docentes y autoridades conocen o no ciertos protocolos como el de Reanimación Cardiopulmonar en niños menores de un año, a lo cual el grupo de docentes que sí conoce fue del $76 \%$ y de los que no fue del $24 \%$. Se planteó un interrogante similar al conocimiento del RCP, pero en niños mayores de un año; la respuesta positiva fue mayor a la anterior pues el $83 \%$ de docentes tiene un conocimiento de las maniobras de RCP y el
$17 \%$ no tiene un conocimiento acerca de la misma. Como parte de las maniobras básicas dentro de los protocolos de primeros auxilios, existen las maniobras de reanimación cardio pulmonar; a esta pregunta el $69 \%$ de los encuestados dijo si conocer sobre la maniobra y 31 \% dijo no conocer. En relación a la misma maniobra, pero en mayores de un año, el $56 \%$ si la conoce y sabe cómo aplicarla y el $44 \%$ desconoce de la misma.

\section{Figura 2}

Porcentaje promedio de conocimientos de primeros auxilios en función de preguntas prácticas

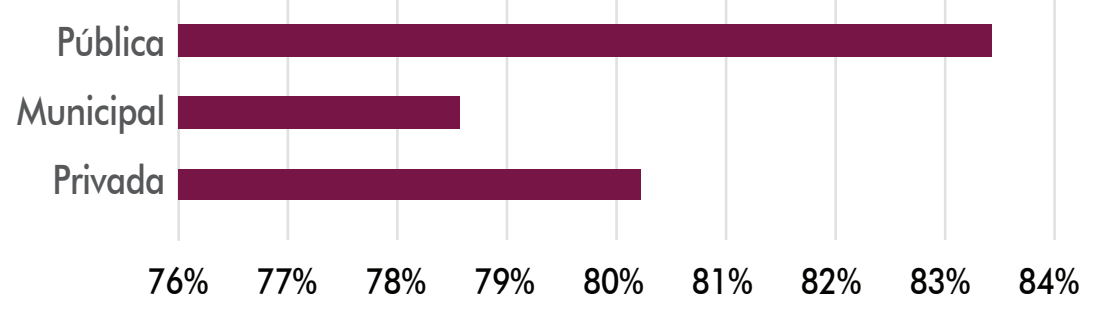

Nota. Obtenido de encuesta.

La Figura 2 muestra promedio y porcentajes de conocimientos prácticos de primeros auxilios de los docentes y autoridades de las instituciones escolares investigados, para enfrentar o actuar ante eventos cotidianos que pueden surgir; así: Del universo total de docentes de las instituciones públicas, el 83 \% tienen conocimiento de primeros auxilios de forma práctica; se observa que de la población total de los funcionarios de las instituciones municipales, el $79 \%$ conocen de forma práctica primeros auxilios; finalmente deja entrever que del total de docentes y autoridades que laboran en entidades privadas el $80 \%$ de profesionales cuentan con este tipo de conocimientos. 
Nivel de conocimiento de protocolos de primeros auxilios en docentes de educación inicial

y preparatoria de instituciones educativas de la ciudad de Quito - María Teresa Murillo,

Erika Marlene Vargas, Janeth del Pilar López • VÍNCULOS-ESPE (2021) VOL.6, No.3: 47-66

La Figura 2 presenta los conocimientos prácticos en primeros auxilios, que los docentes y autoridades tienen. Se planteó la interrogante, ¿̇sí conocían qué hacer ante ciertos tipos de accidentes que pueden llegar a ocurrir dentro de las instalaciones educativas? Por lo que, el grupo de 54 docentes indicaron una respuesta positiva, así: el $15 \%$ conoce como actuar frente a una asfixia y heridas; en relación a las fracturas, el $11 \%$; en caso de una luxación, el $6 \%$; en caso de una quemadura de cualquier grado, el $5 \%$; en caso de convulsiones, el $6 \%$; en caso de intoxicación, el $13 \%$; en caso de electrocución, el 1\%; en casos de desmayos, el 15 $\%$; en casos de sangrado nasal o epistaxis, el $4 \%$; en caso de esguinces, el 6\%; en caso de politraumatismos, el 1\%, y en caso de paros cardiorrespiratorio, el $2 \%$. Un estudio similar realizado por Quispe (2019) evalúa los conocimientos de los docentes y coinciden en las tipologías de accidentes en los que se tienen que poner en práctica los primeros auxilios; así se citan asfixia por atragantamiento y heridas; y, deja entrever su preocupación por existir deficiencia en procedimientos como las quemaduras, desmayos, etc. (p.43)

Dentro del instrumento aplicado se consideró como parte de los protocolos de primeros auxilios, el contar con al menos un botiquín para ser utilizado en caso de una emergencia. El $89 \%$ contestó que sí posee y el 11 \% dijo no contar con un botiquín. En relación a la interrogante, se planteó una lista de insumos médicos considerados necesarios dentro del botiquín de primeros auxilios, dentro de los cuales el $5 \%$ tiene guías telefónicas de centros médicos, hospitales cercanos o de emergencia; el $13 \%$ tiene curitas; $12 \%$ gasas, $12 \%$ algodón, $5 \%$ guantes quirúrgicos, 3 \% mascarillas quirúrgicas, 1 \% abate lenguas, $3 \%$ vendas de varios tamaños, $11 \%$ alcohol antiséptico, $12 \%$ agua oxigenada, $4 \%$ paracetamol, $0 \%$ termómetro, $1 \%$ férulas, 0 $\%$ vendajes triangulares, $4 \%$ pinzas, $0 \%$ esparadrapo, $0 \%$ apuntes de alergias o enfermedades de los niños, tipo de sangre y medicamentos que usan, y un $0 \%$ loratadina.

Si se compara con lo que sugiere Seattle Childrens (2020) se observa que existen insumos similares que consideran como: el directorio, vendajes, curitas, gasa, cintas adhesivas, cotonetes, agua, jabón, toallas antisépticas, termómetro, guantes, pinzas, tijeras paño, y otros insumos no están incorporados como acetaminofén, ibuprofeno, aspirina, medicina oral, soluciones con electrolitos, cremas con hidrocortisona, gel de aloe, agua salina, loción con calamina; se puede inferir a que es porque el procedimiento de primeros auxilios es realizado por docentes y sus conocimientos son elementales de los primeros auxilios.

\section{Figura 3}

Promedio de los conocimientos en primeros auxilios preguntas prácticas en porcentaje

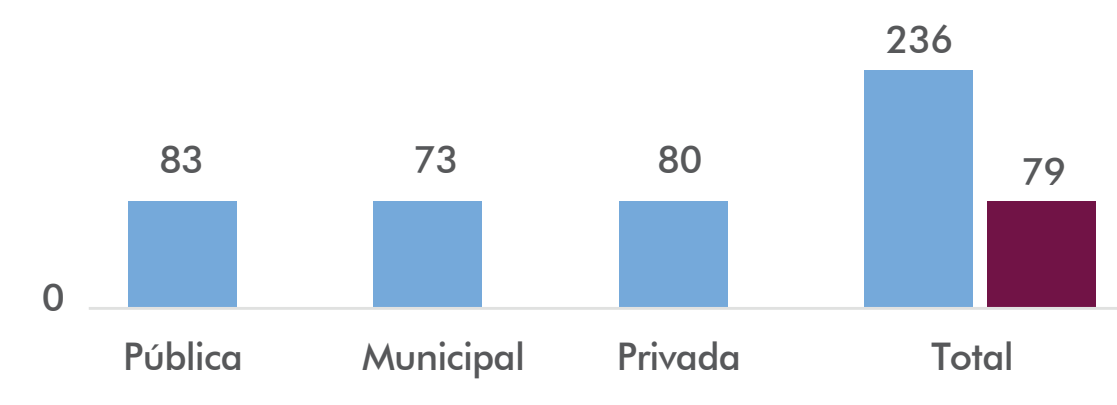

$\%$

- Promedio

Nota. Obtenido de encuesta. 
La Figura 3 presenta resultados en función del promedio de conocimientos prácticos en primeros auxilios con preguntas prácticas; así, reflejan que el 79 \% de los profesionales de los establecimientos educativos tiene conocimientos de primeros auxilios con base en preguntas prácticas. En relación a este tema, existe un estudio realizado por Zuriñe \& Velasco (2017) aplicó a 2 grupos de profesionales un test, uno de los grupos superó el test propuesto con un porcentaje del 68,7 \%, sin embargo, se determinó que existen carencias de conocimiento en cada una de las áreas evaluadas. Si se compara el resultado obtenido en el estudio propuesto y el estudio realizado por Zuriñe \& Velasco (2017), se puede evidenciar que los porcentajes que reflejan conocimiento sobre primeros auxilios superan el $50 \%$; pues estos valores son $79 \%$ y $68.7 \%$ respectivamente, mismos que dejan entrever que existe un porcentaje que desconoce los procedimientos de primeros auxilios.

\section{Figura 4}

Promedio de los conocimientos en primeros auxilios preguntas teóricas

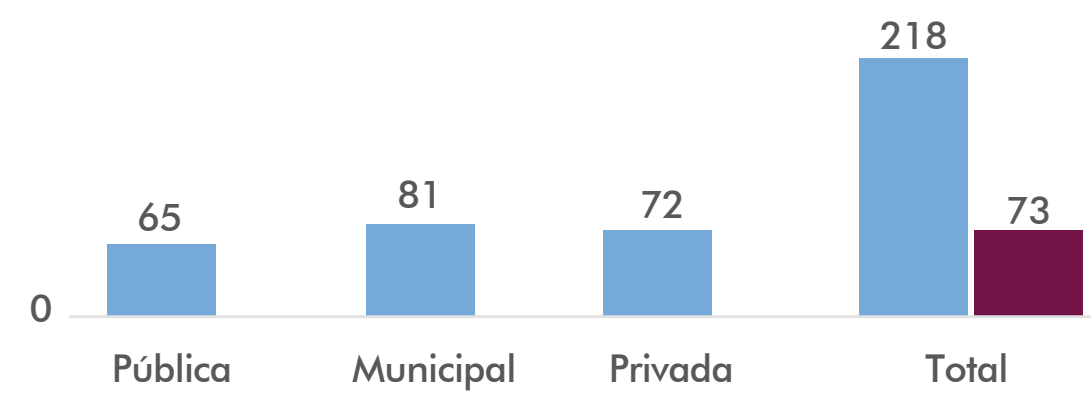

a Promedio

Nota. Obtenido de encuesta.
La Figura 4 establece promedio $73 \%$ de profesionales de las Instituciones educativas publicas, municipales y privadas tienen conocimientos teóricos en primeros auxilios. Si se relaciona, este resultado con los conocimientos prácticos existe una diferencia de $6 \%$, por lo que se puede inferir que esta diferencia se presenta cuando los docentes deben intervenir para dar respuesta a la emergencia, sin conocer si esta intervención es la adecuada y oportuna. Tal como lo sostiene Cantos, (2018), los docentes necesitan tener conocimientos específicos y generales de primeros auxilios por lo que necesario "apropiarse no solo de los conocimientos propios de su disciplina sino además de aquellas cosas que resultan importantes, prácticas y oportunas en su quehacer en el aula..." (p.48); con lo que se confirma la necesidad de capacitación a los docentes que tengan conocimientos teóricos y prácticas

\section{DISCUSIÓN}

La población involucrada en la lnvestigación son 54 profesionales de la educación, de los cuales 42 son docentes y 12 se desempeñan como directivos de las instituciones educativas, públicas, municipales y privadas. En relación con los factores asociados al conocimiento en primeros auxilios, el $98 \%$ conocen de los procedimientos de primeros auxilios y un reducido porcentaje del $2 \%$ aceptaron no conocer cómo proceder ante una emergencia. La aplicación del instrumento a los y las profesiones encuestadas proyecta la siguiente información: que existe un porcentaje de $79 \%$ de profesionales que conocen de forma práctica primeros auxilios; así mismo, que un $73 \%$ de los y las encuestadas señalan tener conocimientos teóricos 
en primeros auxilios; lo significa que existe un porcentaje de docentes que poseen conocimientos teóricos y prácticos.

Así mismo del presente estudio se desprende que las y los docentes no poseen una capacitación integral con respecto a primeros auxilios; pues Cantos (2018) en un estudio señala que:

$40 \%$ de los accidentes ocurre en educación básica, siendo los más pequeños quienes están más expuestos a los eventos que pueden ocurrir en cualquier momento y lugar, y es indispensable que los funcionarios en las escuelas, liceos y colegios puedan tomar diversas medidas antes de que llegue la atención médica, a fin de estabilizar a la persona que vive la situación de emergencia y prepararla para el tratamiento y traslado oportuno. (p.48)

Se desprende que recibieron capacitación el 4\% recibió su capacitación en el año 2004; otro $4 \%$ fue capacitado en el año 2016; el $13 \%$ en el año 2018; el $8 \%$ en el año 2019; y el $71 \%$ no especifica en que año recibió la capacitación. Considerando la información señalada se cita a los autores Aldrete, Mireles, Mendoza, \& Aranda (2004), que determinaron la existencia de profesionales que nunca han participado en cursos específicos de primeros auxilios; otros que sí los tomaron fue por medio del programa de Seguridad y Emergencia Escolar.

Se considera entonces que la limitada capacitación en primeros auxilios de las y los docentes es una constante, por lo que es indispensable que la población total de docente de las instituciones educativas acceda a eventos de capacitación en todas las áreas de los primeros auxilios con la finalidad de prevenir y actuar oportunamente. Las autoridades deben hacer énfasis en capacitar y preparar a las/os docentes, para que actúen de forma oportuna frente a los accidentes en las instalaciones educativas, conforme establezca el protocolo intervención conforme el Plan de Emergencias, propuesto desde el Mineduc. El instrumento aplicado enfocó su atención a saber si los docentes y autoridades conocían sobre la maniobra de Heimlich o RCP (Reanimación Cardiopulmonar), y si sabían el protocolo a seguir. El $43 \%$ contestó que sí y el $57 \%$ dijo no saber qué hacer. Frente a intervenciones con niños/as con discapacidad y enfermedades catastróficas respondieron el 41 \% que sí saben qué hacer, y el 59 \% lo desconoce.

En cuanto al nivel de conocimientos de docente y autoridad en de primeros auxilios, el $54 \%$ indicaron que su conocimiento es bueno, el $28 \%$ indicó tener un conocimiento malo; el grupo de docentes y autoridades encuestados, tiene deficientes conocimientos de primeros auxilios; pese a que existen guías proporcionadas por el Mineduc; es necesario que se enfatice en el fortalecimiento de conocimiento en primeros auxilios; la preparación debe ser constante para atender emergencias dentro de las aulas de clase e instalaciones educativas, a fin de salvaguardar la vida de las y los niños. Se precisa fortalecer las estrategias para contar con personal capacitado de las instituciones, tal como precisan Alderete, ireles, Mendoza, \& Aranda (2004) puesto que refuerzan la necesidad de contar con recursos suficientes en las instituciones educativas para que fortalezcan su plan de intervención en primeros auxilios; consideran importante fortalecer la cultura preventiva y de autocuidado frente a los accidentes en los primeros años de la vida escolar. 


\section{CONCLUSIONES}

La muestra estipulada para el presente trabajo investigativo en relación al conocimiento de primeros auxilios, fue del $46 \%$ de profesionales de las instituciones públicas, el $30 \%$ municipales, y el $24 \%$ privadas que colaboraron facilitando la información.

En conocimiento del tema de primeros auxilios, el $98 \%$ de los encuestados señalaron que conocían los procedimientos de primeros auxilios y un $2 \%$ aceptaron no conocer cómo proceder ante una emergencia.

Los conocimientos que poseen los profesionales de las instituciones educativas públicas, municipales y privadas son deficientes e inconclusos en cuanto protocolos de primeros auxilios en razón de que existen datos que señalan que no recibieron capacitación en su totalidad; así el $4 \%$ recibió su capacitación en el año 2004; otro $4 \%$ fue capacitado en el año 2016; el $13 \%$ en el año 2018 , el $8 \%$ en el año 2019 y el 71 \% no especifica en que año recibió la capacitación.

Es necesario la formación y capacitación docente en la aplicación de un modelo de simulación in situ, que permita poner en práctica los conocimientos de primeros auxilios en simulacros.

Se establece que las instituciones educativas, deben contar con recursos financieros que les permita implementar todos los protocolos de emergencia, que incluye la implementación de botiquines.

Se evidenció la necesidad de contar con un Plan de Emergencia para enfrentar las múltiples eventualidades; puesto que, el 98\% de los docentes y autoridades conocen las directrices desde el MINEDUC, y apliquen el instructivo para la elaboración de un Plan de Emergencias.

\section{AGRADECIMIENTO}

Agradecemos a cada uno de los docentes y autoridades de las instuciones educativas públicas, municipales y privadas, que facilitaron con su predisposición para la recolección de información, y consecuentemente la realización investigación de esta tematica. De igual forma, es importante reconocer la labor de nuestras familias, por el apoyo como pilares fundamentales durante todo este proceso.

\section{REFERENCIAS}

Aldrete, M., Mireles, M., Mendoza, P., \& Aranda, C. (2004). La actitud de los profesores de educación primaria ante los primeros auxilios. 19-24.

Cagua, F. (Septiembre de 2019). Guía de sensibilización para ayudar a la inclusión de estudiantes con enfermedades catastróficas (cáncer) dirigidas al claustro docente de la unidad educativa siglo XXI Luis Vargas Torres. Tesis. Santo Domingo, Santo Domingo de los Tsachillas, Ecuador: Pontificia Universidad Católica del Ecuador.

Cantos, E. (2018). Los primeros auxilios en la enseñanza de los docentes. Polo Conocimiento, 46-53. 
Nivel de conocimiento de protocolos de primeros auxilios en docentes de educación inicial

y preparatoria de instituciones educativas de la ciudad de Quito - María Teresa Murillo,

Erika Marlene Vargas, Janeth del Pilar López • VÍNCULOS-ESPE (2021) VOL.6, No.3: 47-66

Chachapoya. (2019). Registros administrativos internos Unidad Educativa Municipal Oswaldo Lombeyda periodo académico 2018-2019. Quito, Pichincha, Ecuador: Unidad Educativa Municipal Oswaldo Lombeyda .

Childrens Settle. (2020). Botiquin de primeros auxilios dentro y fuera del hogar. Material educativo para la comunidadFirst Aid Kits for Home and Away / [Spanish], 1-2.

Cueva, A. (2015). Repositorio de la universidad de San Francisco de Quito. Obtenido de http://repositorio.usfq. edu.ec/bitstream/23000/4482/1/1 13597.pdf

Delgado, V., \& Espinoza, A. (2019). Repositorio Digital Universidad Laica de Guayaquil. Obtenido de http:// repositorio.ulvr.edu.ec/handle/44000/2590

Ecuador, M. d. (2014). Ministerio de educación del Ecuador. Obtenido de https://educacion.gob.ec/wp-content/ uploads/downloads/2016/03/CURRICULO-DE EDUCACION-INICIAL.pdf

Ecuador, M. d. (2020). Ministerio de Educación. Obtenido de https://educacion.gob.ec/wp-content/plugins/ download-monitor/download.php?id=15713

Ecuatoriana, C. R. (febrero de 2017). Cruz Roja Ecuatoriana. Obtenido de http://www.cruzroja.org.ec/index.php/ featured-video/83-primeros-auxilios

Fernández, M., \& Fernández, I. (2015). Primeros Auxilios de niños y bebés, actuación y tratamiento. Vigo, España: Ilustraciones interiores. "Manual de Cruz Roja de Primeros Auxilios".
Gómez, H; Martínez, A; Casal, C; Ballestar, L. (2015) Proyecto de investigación: Implantación de un programa de primeros auxilios para el alumnado de secundaria de Xirivella (Tesis Universidad de Valencia). Biblioteca Las Casas.

Hernández, M. (2015). El concepto de discapacidad: de la enfermedad al enfoque de derechos. Revista CES Derecho, 6(2), 46-59.

Laura, E., Lorena, G., \& Irma, H. (Enero de 2017). Manual Básico de Primeros Auxilios. Guadalajara, México, Mexico: Unidad Interna de Protección Civil.

LOEl. (Agosto de 2015). Ministerio de Educación del Ecuador. Obtenido de https://educacion.gob.ec/wpcontent/uploads/downloads/2017/02/Ley Organica de Educacion Intercultural LOEl codificado.pdf

Martín, A. (2015). Primeros Auxilios dirigidos al personal docente del ámbito escolar. Revista Educativa Hekademos, $17,85-92$.

Maza, A., \& Maza, B. (2019). Primeros Auxilios (Segunda Edición ed.). España: Editorial Síntesis S.A.

Organización Mundial de la Salud. (2011). Informe Mundial sobre Discapacidades. Ginebra-Suiza: Editorial OMS.

Organización Mundial de la Salud. $\quad\left(\begin{array}{llll}2 & 0 & 2 & 0\end{array}\right)$. ENFERMEDADES CRÓNICAS. Obtenido de https://www. who.int/topics/chronic diseases/es/

Organización Mundial de la Salud. (2020). Reducción de la mortalidad en la niñez. Ginebra: OMS Organización Mundial de Salud WHO. 
Oña, W. (2016). Nivel de conocimientos que poseen los profesores de educación física de los colegios urbanos en Ibarra para brindar primeros auxilios. Tesis para titulación. Ibarra, Imbabura, Ecuador: Universidad Técnica del Norte.

Páez, S. (2015). Reintegración del niño con cáncer en la escuela. Gaceta Mexicana de Oncología, 16(6), 342345.

Quispe, M. (2019). Conocimiento de primeros Auxilios en docentes de la Institución educativa prmiaria María Auxiliadora de Puno 2018. Tesis previo la obtención de la licenciatura de Enfermería. Puno, Perú.

Salvatierra, O. (Noviembre de 2017). LOS PRIMEROS AUXILIOS EN LA ATENCIÓN DE LOS ACCIDENTES ESCOLARES EN NIÑOS Y NIÑAS DE EDUCACIÓN INICIAL. TESIS PREVIO A LA OBTENCIÓN DEL TITULO DE LICENCIADA EN CIENCIAS DE LA EDUCACIÓN MENCIÓN: EDUCADORES DE PÁRVULOS. Guayaquil, Guayas,
Ecuador: UNIVERSIDAD DE GUAYAQUILFACULTAD DE FILOSOFÍA, LETRAS Y CIENCIAS DE LA EDUCACIÓN.

Singh, L., Espinosa, M., Aties, M., \& García, Y. (s.f.). Accidentes o lesiones no intencionales en la infancia. (U. d. Médicas, Ed.) Revista de información científica, 95(6), 1019-1028.

Socota, D. (2014). ESTADO DEL ARTE ACCIDENTES ESCOLARES EN INSTITUCIONES EDUCATIVAS. Tesis previa a la obtención de grado de enfermería. Chía, Cundinamarca, Colombia: Universidad de la Sabana.

Trujillo, Y. (02 de 01 de 2020). Bebé en guardería de Quito falleció; la madre pide que el centro sea cerrado. Obtenido de El Comercio: https://www.elcomercio.com/ actualidad/madre-bebe-muerte-guarderia-quito.html

Zuriñe, G., \& Velasco, Z. (2017). Análisis del Grado de Formación en Primeros Auxilios del Profesorado en Activo de Educación Infantil y Primaria. Formacion Universitaria, 1-1 


\section{BIOGRAFÍA DE LOS AUTORES}

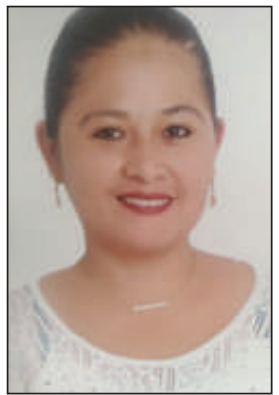

\section{MARÍA TERESA MURILLO LOJÁN}

Egresada de la Carrera de Educación Inicial en la Universidad de las Fuerzas Armadas ESPE. Con experiencia laboral como docente auxiliar de Inicial y Preparatoria en instituciones privadas, municipal y del estado de la ciudad de Quito, Ecuador. Intereses de investigación relacionados a temas de Estimulación Temprana e Inclusión Educativa.

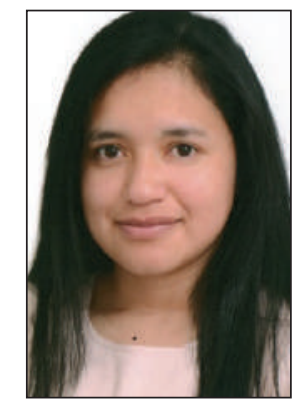

ERIKA MARLENE VARGAS FUERTES

Egresada de la Carrera de Educación Inicial en la Universidad de las Fuerzas Armadas ESPE. Experiencia laboral como auxiliar docente de Inicial | y || en instituciones públicas y privadas de la ciudad de Quito. Inclinación investigativa en temas relacionados a las necesidades educativas especiales infantiles y desarrollo del lenguaje.

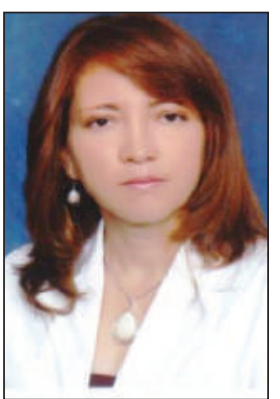

DRA. JANETH DEL PILAR LÓPEZ SEVILLA

Docente Departamento de Ciencias Humanas y Sociales Universidad de las Fuerzas Armadas ESPE. Estudios realizados: Especialista en Neonatología, Universidad San Francisco de Quito, 2019.Especialista en Pediatría, Universidad Central del Ecuador, 2003, Doctor en Medicina, Universidad de Cuenca, 1996. Experiencia Profesional: Médico Tratante del Servicio de Neonatología, Hospital Pediátrico Baca Ortiz, Médico Pediatra Tratante Hospital San Francisco de Quito, Médico Pediatra de Emergencias del Hospital de Los Valles. 